\title{
A NEW OMITTING TYPES THEOREM
}

\author{
CHARLES STEINHORN ${ }^{1}$
}

\begin{abstract}
An omitting types theorem for countable models of superstable theories containing an infinite set of indiscernibles is proved. Various corollaries and applications are given.
\end{abstract}

1. One of the most useful ways that the model theorist has for controlling models is to attempt to specify the element types that models realize and omit. In this paper we present an omitting types theorem which seems well suited to applications to superstable theories, and whose proof relies on techniques from stability theory. For $\omega$-stable theories, the existence of prime models over arbitrary subsets allows one to omit many types, but the existence of such models is not guaranteed in superstable theories. It is hoped that the technique developed here allows one to obtain some of the consequences of the existence of prime models over arbitrary subsets when such models are not available.

In $\S 2$, the basic omitting types theorem is proved and some counterexamples to various strengthenings are given. Some applications and corollaries of the theorem are presented in $\S 3$. The notation used throughout is standard.

2. We assume the reader is familiar with some of the basic notions of stability theory. Any unexplained terms may, of course, be found in [4, III, §§1-3]. The main result of this section is

THEOREM 2.1. Let $T$ be superstable and $\Re$ a countable model of $T$ containing an infinite set of indiscernibles. If $p(\bar{x}, \bar{m})$ is a type with a finite sequence of parameters $\bar{m} \in \mathfrak{N}$ that is omitted in $\mathfrak{R}$, then there is an $\mathfrak{x} \mathfrak{N}$ that also omits $p(\bar{x}, \bar{m})$.

Before proving Theorem 2.1, we shall have to avail ourselves of some preliminaries. All results may be found in [4, III, §§1-3].

Definition 2.2. Let $T$ be stable, $C \subseteq \mathfrak{N} \vDash T$ and $I \subseteq \Re$ be an infinite sequence (hence set) of indiscernibles. The average type of $I$ over $C$, denoted by $\operatorname{Av}(I, C)$, is $\left\{\varphi(x, \bar{c}): \bar{c} \in C \wedge|\{i \in I: \Re \vDash \varphi(i, \bar{c})\}| \geqslant \aleph_{0}\right\}$.

The lemma below justifies the terminology employed in the definition.

Lemma 2.3. For stable $T$, the average type of $I$ over $C$, for any $I$ and $C$, is a consistent and complete set of formulas.

Received by the editors January 19, 1983.

1980 Mathematics Subject Classification. Primary 02H05, 02H13.

'Partially supported by a grant from NSERC of Canada.

(C)1983 American Mathematical Society $0002-9939 / 83 \$ 1.00+\$ .25$ per page 
Lemma 2.4. Let $T$ be superstable, $A \subseteq \Re \vDash T$ and $I \subseteq \Re$ a set which is indiscernible over $A$. If $B \subseteq \mathfrak{N}$ is finite, then there exists a finite $J \subseteq I$ so that $I \backslash J$ is indiscernible over $A \cup B \cup J$.

Now we can proceed with the proof of Theorem 2.1.

Proof of Theorem 2.1. Let $q(x)$ be the average type of $I$ over $\Re$ in the language $L(\mathfrak{N})$ having constants for each $m \in \mathfrak{N}$. Let $d$ be a new constant symbol and $T^{*}=q(d)$ the resulting complete theory in $L(\Re \cup\{d\})$. By the ordinary omitting types theorem it suffices to show that $T^{*}$ locally omits $p(\bar{x}, \bar{m})$ (observe that $d \neq m \in T^{*}$ for each $\in \mathfrak{T}$ ).

We suppose $T^{*}$ does not locally omit $p(\bar{x}, \bar{m})$ and obtain a contradiction. That is, let $\theta\left(\bar{x}, d, \bar{m}^{\prime}\right)$ be consistent with $T^{*}$, where $\bar{m}^{\prime} \in \Re$, but assume that $T^{*} \vdash$ $\theta\left(\bar{x}, d, \bar{m}^{\prime}\right) \rightarrow \sigma(\bar{x}, \bar{m})$ for each $\sigma(\bar{x}, \bar{m}) \in p(\bar{x}, \bar{m})$. It follows that for each $\sigma(\bar{x}, \bar{m})$ $\in p(\bar{x}, \bar{m})$, the formula $(\forall \bar{x}) \theta\left(\bar{x}, y, \bar{m}^{\prime}\right) \rightarrow \sigma(\bar{x}, \bar{m})$ is in the average type of $I$ over T (as a type in the variable $y$ ). Since $B=\left\{\bar{m}, \bar{m}^{\prime}\right\}$ (considered as a set of elements of $\Re$ ) is finite, by Lemma 2.4 , there is some finite $J \subseteq I$ so that $I \backslash J$ is indiscernible over $B \cup J$. Therefore, for any $i \in I \backslash J$, and $\sigma(\bar{x}, \bar{m}) \in p(\bar{x}, \bar{m})$,

$$
\mathfrak{R} \vDash(\forall \bar{x}) \theta\left(\bar{x}, i, \bar{m}^{\prime}\right) \rightarrow \sigma(\bar{x}, \bar{m}) .
$$

This would imply that any sequence of elements in $\mathfrak{T}$ satisfying $\theta\left(\bar{x}, i, \bar{m}^{\prime}\right)$ - of which there must be one, since $(\exists \bar{x}) \theta\left(\bar{x}, y, \bar{m}^{\prime}\right)$ is in the average type of $I$ over 爪_realizes $p(\bar{x}, \bar{m})$. Since $p(\bar{x}, \bar{m})$ was to have been omitted in $\Re$, we have a contradiction. This complets the proof of 2.1.

By the ordinary Extended Omitting Types Theorem, we immediately have the following corollary.

COROLlaRY 2.5. Let $T$ be superstable and $\mathfrak{N}$ a countable model of $T$ containing an infinite set of indiscernibles. If, for each $k<\omega, p_{k}\left(\bar{x}_{k}, \bar{m}_{k}\right)$ is a type with a finite sequence of parameters $\bar{m}_{k} \in \mathfrak{R}$ that is omitted in $\mathfrak{R}$, then there is an $\Re \nsucceq \mathfrak{T}$ that omits each $p_{k}\left(\bar{x}_{k}, \bar{m}_{k}\right)$.

Before stating the next result, we must make additional definitions. They are standard in the literature.

Definition 2.6. Let $T$ be stable, $\Re \vDash T, A \subseteq \Re$. A type $p \in S(A)$ is stationary if for any $B \supseteq A, p$ has a unique nonforking extension to an element of $S(B)$. A sequence of elements $I=\left\{i_{j}: j<\xi\right\}$ is a Morley sequence based on the stationary $p \in S(A)$ if for each $j<\xi, i_{\xi}$ satisfies the unique nonforking extension of $p$ to $S\left(A \cup\left\{i_{k}: k<j\right\}\right)$.

It is well known, cf. [4, III, §4], that if $T$ is superstable and $I$ is an infinite set of indiscernibles, then there is no loss of generality in assuming that $I$ is an infinite Morley sequence based on some stationary $p \in S(A)$ for some finite $A$. It seems natural to ask if Theorem 2.1 can be strengthened by requiring only that $\mathfrak{T}$ contain arbitrarily long finite Morley sequences based on the same stationary $p \in S(A)$, 
$A \subseteq \Re$ and $A$ finite. Indeed,

COROLlaRy 2.7. Let $T$ be superstable, $\mathfrak{N} \vDash T$, and $\mathfrak{N}$ countable. Suppose also that OR contains arbitrarily long finite Morley sequences based on some stationary $p \in S(A)$, $A \subseteq \mathcal{O}$ and $A$ finite. Then, if $p(\bar{x}, \bar{m})$ is a type with a finite sequence of parameters $\bar{m} \in \mathfrak{N}$ that is omitted in $\Re$, there is an $¥ \Re$ that also omits $p(\bar{x}, \bar{m})$.

After we had proved this, it was pointed out to us by A. Pillay that the strengthening is illusory. The lemma below shows that 2.7 is no more than 2.1 in disguise.

Lemma 2.8. Let $T$ be superstable, $\Re \vDash T$ and $p \in S(A), A \subseteq \Re$ and $A$ finite, be stationary. If $\mathfrak{N}$ contains arbitrarily long finite Morley sequences based on $p$, then $\Re$ contains an infinite Morley sequence based on p.

Proof. For each $n<\omega$, let $I_{n}$ be a Morley sequence in $\Re$ of length $n$ that is based on $p$. We show that we may extend any $I_{N}$ to a Morley sequence in $\Re$ with $N+1$ elements. By repeating the argument, the lemma will be proved.

In $[4, \mathrm{~V}, \S 3]$, it is shown that if $T$ is superstable, then to any type $p \in S(B)$, where $B$ is arbitrary, there can be assigned a unique natural number $n$, called the weight of $p$ with the property that:

For any $\bar{c}$ satisfying $p$ and any sequence $\left\langle\bar{d}_{j}: j\langle K\rangle\right.$, if for all $k<K, t\left(d_{k}, A \cup\left\{\bar{d}_{j}: j \neq k\right\}\right)$ does not fork over $A$, and for all $k<K, t\left(\bar{d}_{k}, A \cup\{\bar{c}\}\right)$ forks over $A$, then $K \leqslant n$.

Let the weight of $t\left(I_{N}, A\right)=n$.

It follows that there is a $c \in I_{n+1}$ so that $t\left(c, A \cup I_{N}\right)$ does not fork over $A$. Since $c$ realizes $p$, we have that $I_{N} \cup\{c\}$ is a Morley sequence.

However, if $\Re$ as in 2.7 merely contains arbitrarily long finite sequences of indiscernibles whose $n$-types are the same, for each $n$, we do not know if the corresponding theorem holds. If the $n$-types are permitted to be different, then the corresponding version of the theorem is false, even if $T$ is $\omega$-stable: consider the theory of an equivalence relation having a class of size $n$, for each $n<\omega$.

If $T$ is unstable, then there is a counterexample even to the analogue of 2.1 , which was found by M. Kaufmann.

ExAmple 2.9. Let $\Re=\langle M, W, U, V,<, h\rangle$, where $W, U$ and $V$ are disjoint unary predicates whose union is $M,<$ is a linear order of type $\omega$ on $W, V=\{g: W \rightarrow U: g$ is a bijection and $\left\{x: g(x) \neq g_{0}(x)\right\}$ is finite $\}$, where $g_{0}: W \rightarrow U$ is a fixed bijection, and $h: V \times W \rightarrow U$ is given by $h(g, x)=g(x)$.

It is not difficult to see that $U$ is a set of indiscernibles in $\Re$. We claim that the type $p(x)=\{W(x)\} \cup\{$ " $x>n$ ": $n<\omega\}$, where " $x>n$ " abbreviates the parameter-free formula saying that $x$ is bigger than the $n$th element of $W$ under $<$, cannot be omitted in any proper elementary extension of $\mathscr{N}$ although it clearly is omitted in গ⿰. 
Suppose $\Re>\Re$ and $\Re$ omits $p$. We show that $\Re=\Re$. First, $W^{\Re}=W^{\Re}$ since $p$ is omitted. Now fix $g \in V^{\Re}$. By elementarity,

$$
\mathcal{X} \vDash \forall x[U(x) \rightarrow \exists y(W(y) \wedge h(g, y)=x)] .
$$

It follows that $U^{\Re}=U^{\Re}$. Finally, to see that $V^{\Re}=V^{\Re}$, we observe that the statements below are true in $\Re$, and thus in $\Re$ :

(i) $\forall x \forall y \exists z[W(z) \wedge \forall u[(W(u) \wedge z<u) \rightarrow h(x, u)=h(y, u)]]$.

(ii) $\forall x \forall y[\forall z(W(z) \rightarrow h(x, z)=h(y, z)) \rightarrow x=y]$.

If $f \in V^{\Re}$ and $g \in V^{\Re}$, then, since $W^{\Re}=W^{\Re}$, (i) implies that $f$ and $g$ differ only on a finite set. But then there is some $f^{\prime} \in V^{\mathcal{N}}$ so that $\mathscr{R} \vDash h\left(f^{\prime}, m\right)=h(f, m)$ for all $m \in W^{\mathfrak{R}}=W^{\Re}$. From (ii) we conclude that $f^{\prime}=f$ and $f^{\prime} \in \mathfrak{N}$, as desired.

In the case that $T$ is stable but not superstable, we can use the model constructed in [3] to find a type over finitely many parameters that is omitted in the model but is implied by the average type of a set of indiscernibles in the model. Thus the method of proof of Theorem 2.1 will not apply. Furthermore, although we do not have a stable, not-superstable example analogous to 2.9 , we do know that the model built in [3] admits a countable collection of types all over a fixed finite set of parameters that cannot all be omitted in a proper elementary extension.

Lastly, we note the extension below of Theorem 2.1 for uncountable languages. It is modeled upon the ordinary $\kappa$-Omitting Types Theorem (cf. [1]).

THEOREM 2.10. Let $T$ be superstable and $|T|=\kappa$. Let $\mathfrak{N} \vDash T$ be compact (i.e., $\kappa$-compact and of power $\kappa)$ and contain a set of indiscernibles of power $\kappa$. If $p(x, A)$ is a type over $A$, where $A=\left\{a_{\xi}: \xi<\alpha\right\} \subseteq \mathfrak{K}, \alpha<\kappa$, that is omitted in $\mathfrak{K}$, then there is an $\Re \nsucceq$ গ that also omits $p$.

We omit the proof. It utilizes lemmas similar to those needed to establish Theorem 2.1 , which can be found in $[4, \mathrm{III}, \S \S 1-3]$.

3. In this section we present some extensions and applications of the results of $\S 2$. The emphasis here is on elementarily extending countable models in such a way as to preserve properties such as homogeneity. As will be seen, under most conditions the extension may be made uncountable. This was exploited by Buechler [0] in setting Kueker's conjecture for superstable theories. The theorems below are not, of course, intended to be exhaustive or definitive, but merely suggestive of how our results might be used.

Since the elementary extension obtained in 2.1 , or 2.5 , still satisfies the hypothesis of the theorem, we may iterate the process $\omega_{1}$ times to obtain

THEOREM 3.1. Let $T$ be superstable, $\Re \vDash T$, $\Re$ countable and containing an infinite set of indiscernibles. If, for each $k<\omega, p_{k}\left(\bar{x}_{k}, \bar{m}_{k}\right)$ is a type over the finite sequence of parameters $\bar{m}_{k} \in \mathfrak{N}$ that is omitted in $\Re$, there is an $¥ \Re,|\Re|=\boldsymbol{\aleph}_{1}$, that omits each $p_{k}$.

It is also clear that at each stage in the construction of $\Re$ we may choose to omit an additional countable collection of types all of which are omitted in the model built at that stage in the construction. 
It is unknown to us if models of power greater than $\aleph_{1}$ omitting the types can be built.

We note the following

COROLlary 3.2. Suppose, in addition to the hypotheses of 3.1, that $T$ has only countably many types over $\varnothing$. Then there is an $\Re \nsucceq \Re,|\Re|=\aleph_{1}$, that realizes and omits exactly those types over finitely many parameters from $\Re$ that are realized and omitted in Th itself.

In another direction, one may wonder if the methods of $\$ 2$ may be used to find an elementary extension of a countable model $\mathfrak{T}$ that augments a particular set of indiscernibles and leaves another set fixed. The reader easily can construct examples to see that this is not possible in general. We do, however, have a result, 3.4 , which shows that under suitable hypotheses, some progress on the problem may be made. The result is not entirely satisfactory, though, as it does not permit iteration. Before stating the theorem, we must make some definitions. They are standard and elaborated upon fully in [4, IV, §§1-3].

Definition 3.3. Let $T$ be stable. (i) Let $p \in S(A)$ and $q \in S(B)$. Then $p$ is orthogonal to $q, p \perp q$, if whenever $p^{\prime}$ and $q^{\prime}$ are nonforking extensions of $p$ and $q$, repectively, to any set $C$ containing $A \cup B$, and $t(a, C)=p^{\prime}, t(b, C)=q^{\prime}$, then $t(a, C \cup b)$ does not fork over $C$. (ii) A model $\Re \vDash T$ is $F_{\kappa_{0}}^{a}$-saturated if every strong type over a finite subset of $\Re$ is realized in $\Re$. A model $\Re$ is $F_{\kappa_{0}}^{a}$-prime over a set $A \subseteq \mathfrak{K}$ if it is prime among the $F_{\kappa_{0}}^{a}$-saturated models that contain $A$.

For superstable theories, $F_{\aleph_{0}}^{a}$-prime models exist over any set $A$ (cf. [4, IV, 2.18 and 3.1]). Now we can state the theorem.

THEOREM 3.4. Let $T$ be superstable, $\mathfrak{T} \vDash T$, $\Re$ countable. If $I$ and $J$ are infinite sets of indiscernibles in $\Re$ so that $\operatorname{Av}(I, \Re) \perp \operatorname{Av}(J, \Re)$, there is an $\Re \Varangle \Re$ which realizes $\operatorname{Av}(I, \Re)$ and omits $\operatorname{Av}(J, \Re)$ in addition to a chosen countable set of types over finite subsets $\mathfrak{T}$ that are omitted in $\mathfrak{R}$.

The theorem follows from the next lemma.

LEMMA 3.5. Let $T$ be superstable, $p, q \in S(\mathfrak{T})$, both nonprincipal, and $p \perp q$. Then there is no formula $\theta(\bar{x}, \bar{y}, \bar{m})$ over $\mathfrak{T}$ such that for all $\varphi(\bar{y}) \in q(\bar{y})$,

$$
p(\bar{x}) \vdash \forall \bar{y}(\theta(\bar{x}, \bar{y}, \bar{m}) \rightarrow \varphi(\bar{y})) .
$$

Proof. Suppose not. Let $\mathfrak{K}^{\prime}>\mathfrak{K}$ be $F_{\kappa_{0}}^{a}$-saturated, and let $p^{\prime}$ be the nonforking extension of $p$ over $\Re^{\prime}$. We assert that $\theta(\bar{x}, \bar{y}, \bar{m})$ isolates the nonforking extension, $q^{\prime}(\bar{y})$, of $q$ over TR.

First we show that $\theta(\bar{x}, \bar{y}, \bar{m})$ isolates a type over $\mathfrak{M}^{\prime}$. For if not, then for some $\varphi\left(\bar{y}, \bar{m}^{\prime}\right)$ with $\bar{m}^{\prime} \in \mathfrak{R}^{\prime}$,

$$
p^{\prime}(\bar{x}) \vdash \exists \bar{y}\left[\theta(\bar{x}, \bar{y}, \bar{m}) \wedge \varphi\left(\bar{y}, \bar{m}^{\prime}\right)\right] \wedge \exists y\left[\theta(\bar{x}, \bar{y}, \bar{m}) \wedge \neg \varphi\left(\bar{y}, \bar{m}^{\prime}\right)\right] .
$$

Since $p^{\prime}$ is the nonforking extension of $p$, there must be some $\bar{m}^{\prime \prime} \in \mathfrak{N}$ so that

$$
\exists \bar{y}\left[\theta(\bar{x}, \bar{y}, \bar{m}) \wedge \varphi\left(\bar{y}, \bar{m}^{\prime \prime}\right)\right] \wedge \exists \bar{y}\left[\theta(\bar{x}, \bar{y}, \bar{m}) \wedge \neg \varphi\left(\bar{y}, \bar{m}^{\prime \prime}\right)\right] \in p,
$$

but this is impossible. 
Secondly, we must prove that the type isolated by $\theta(\bar{x}, \bar{y}, \bar{m})$ in $S\left(\mathscr{T}^{\prime}\right)$ does not fork over $\Re$. But just as above, the nonforking of $p^{\prime}$ over $\Re$ implies that if a formula $\varphi\left(\bar{y}, \bar{m}^{\prime}\right) \in q^{\prime}(\bar{y})$, i.e.,

$$
p^{\prime}(\bar{x})+\forall \bar{y}\left(\theta(\bar{x}, \bar{y}, \bar{m}) \rightarrow \varphi\left(\bar{y}, \bar{m}^{\prime}\right)\right),
$$

then there is an $\bar{m}^{\prime \prime} \in \mathfrak{R}$ so that

$$
p(\bar{x}) \vdash \forall \bar{y}\left(\theta(\bar{x}, \bar{y}, \bar{m}) \rightarrow \varphi\left(\bar{y}, \bar{m}^{\prime \prime}\right)\right),
$$

so $\varphi\left(\bar{y}, \bar{m}^{\prime \prime}\right) \in q(\bar{y})$. Thus $q^{\prime}$ must be the nonforking extension of $q$, as desired.

Finally, we see that $q^{\prime}$ must be realized in the model that is $F_{\kappa_{0}}^{a}$-prime over $\Re^{\prime} \cup\{\bar{a}\}$, where $\bar{a}$ realizes $p^{\prime}$. This (cf. $\left[2\right.$, part C]) contradicts the fact that $p^{\prime} \perp q^{\prime}$ (which follows from $p \perp q$ ).

We conclude with applications to homogeneous and recursively saturated models.

THEOREM 3.6. Suppose $T$ is superstable and $|S(\varnothing)|=\boldsymbol{\aleph}_{0}$. Let $\Re \vDash T$, $\Re$ countably homogeneous and containing an infinite set of indiscernibles. Then there is an $N \nsucceq \mathfrak{R}$ which is countably homogeneous and realizes exactly the same types over finite subsets of $\mathfrak{T}$ as does $\Re$.

Of course, we can iterate the process to obtain

COROLlaRy 3.7. Let $\mathfrak{N}$ and $T$ be as in 3.6. Then there is an $\mathfrak{R}^{\prime}>\mathfrak{N}$ that is uncountable, $\omega$-homogeneous and realizes the same types as $\mathfrak{R}$ over finite subsets of 爪.

Proof OF 3.6. By 2.5 there is a countable $\mathfrak{R}_{0} \nsucc \mathfrak{R}$ that realizes exactly the same types over finite subsets of $\Re$ as does $\Re$. To prove the theorem it suffices, by a union of chain argument, to show that if $\left(\mathscr{N}_{0}, \bar{m}_{1}\right) \equiv\left(\mathscr{N}_{0}, \bar{m}_{2}\right)$ and $a \in \Re_{0}$, there is an $\mathfrak{K}_{1}>\mathscr{N}_{0}$ which is countable, realizes the same types over finite subsets of $\mathscr{R}$ as $\Re$, and contains an element $b$ so that $\left(\Re_{1}, \bar{m}_{1} \wedge a\right) \equiv\left(\Re_{1}, \bar{m}_{2} \wedge b\right)$.

Consider the complete type $p(x)$ over the parameters $\bar{m}_{2}$ which is given by $\varphi\left(x, \bar{m}_{2}\right) \in p(x)$ iff $\mathfrak{R}_{0} \vDash \varphi\left(a, \bar{m}_{1}\right)$. Since $\mathfrak{R}$ is homogeneous and $\mathfrak{R}_{0}$ and $\mathfrak{R}$ realize the same types over finitely many parameters from $\Re$, we can construct a type $p^{*}(x) \in S\left(\mathfrak{T}_{0}\right)$ extending $p(x)$ with the additional property that if $\bar{m}^{\prime} \in$ $\mathfrak{T}_{0} \backslash \mathfrak{N}, \bar{m} \in \mathfrak{N}$ and $p_{0}\left(x, \bar{m}^{\prime}, \bar{m}\right)=p^{*}(x) \uparrow L\left(\bar{m}^{\prime}, \bar{m}\right)$, then $p_{0}(x, \bar{y}, \bar{m})$-a type in the variables $x$ and $\bar{y}$-is realized in $\Re$.

Let $d$ be a new constant symbol. We will prove that $T^{*}=p^{*}(d) \cup$ $\operatorname{Th}\left(\mathfrak{N}_{0}, m\right)_{m \in \mathscr{N}_{0}}$ locally omits every type $q(\bar{x}, \bar{m}), \bar{m} \in \mathfrak{N}$, that is omitted in $\Re$. For if not, then for some such $q$, there is a formula $\theta\left(\bar{x}, d, \bar{m}^{\prime}, \bar{m}\right)$ so that

$$
T^{*} \uparrow L\left(d, \bar{m}^{\prime}, \bar{m}\right) \vdash \exists \bar{x} \theta\left(\bar{x}, d, \bar{m}^{\prime}, \bar{m}\right) \wedge \forall \bar{x}\left(\theta\left(\bar{x}, d, \bar{m}^{\prime}, \bar{m}\right) \rightarrow \psi(\bar{x}, \bar{m})\right),
$$

where $\bar{m}^{\prime} \in \mathfrak{M}_{0} \backslash \mathfrak{M}$ and without loss $\bar{m}^{\prime} \supseteq \bar{m}_{0} \wedge \bar{m}_{1}$, and $\psi(\bar{x}, \bar{m}) \in q$.

By construction there are $d^{\prime \prime}, \bar{m}^{\prime \prime} \in \Re$ realizing the type $p_{0}(x, \bar{y}, \bar{m})$, where $p_{0}\left(x, \bar{m}^{\prime}, \bar{m}\right)=p^{*}(x) \uparrow L\left(\bar{m}^{\prime}, \bar{m}\right)$. Therefore

$\operatorname{Th}\left(\left(\Re, d^{\prime \prime}, \bar{m}^{\prime \prime}, \bar{m}\right)\right) \vdash \exists \bar{x} \theta\left(\bar{x}, d^{\prime \prime}, \bar{m}^{\prime \prime}, \bar{m}\right) \wedge \forall \bar{x}\left(\theta\left(x, d^{\prime \prime}, \bar{m}^{\prime \prime}, \bar{m}\right) \rightarrow \psi(\bar{x}, \bar{m})\right)$,

for all formulas $\psi(\bar{x}, \bar{m}) \in q$. But then $q(\bar{x}, \bar{m})$ would be realized in $\Re$, a contradiction. 
Using a similar argument, we obtain

THEOREM 3.8. Let $T$ be superstable and $|S(\varnothing)|=\boldsymbol{\aleph}_{0}$. If $\mathfrak{N}$ is a countable recursively saturated model of $T$ containing an infinite set of indiscernibles, then there exists an $\Re \nsucceq \Re$ ( which can be of power $\aleph_{1}$ ) which is recursively saturated and realizes exaclty the same types over finite subsets of $\mathfrak{K}$ as does $\mathfrak{K}$.

\section{REFERENCES}

0. S. Buechler, Kueker's conjecture for superstable theories, J. Symbolic Logic (to appear).

1. C. C. Chang and H. J. Keisler, Model theory, North-Holland, Amsterdam, 1973.

2. M. Makkai, A survey of basic stability theory, with particular emphasis on orthogonality and regular types (to appear).

3. L. Marcus, A minimal prime model with an infinite set of indiscernibles, Israel J. Math. 11 (1972), 180-183.

4. S. Shelah, Classification theory and the number of nonisomorphic models, North-Holland, Amsterdam, 1978.

Department of Mathematics, McGill University, Montreal, Quebec, Canada H3A 2K6

Current address: Department of Mathematics, Vassar College, Poughkeepsie, New York 12601 